\title{
Herpes Zoster em Pacientes com Lúpus Eritematoso Sistêmico Juvenil
}

\section{Herpes Zoster in Patients with Juvenile Systemic Lupus Erythematosus}

\author{
Paula da Silva Neves ${ }^{(1)}$, Mércia Maria Moreira Facó(2) $^{(2)}$ Adriana Maluf Elias Sallum ${ }^{(3)}$, \\ Lúcia Maria Arruda Campos ${ }^{(3)}$, Alfio Rossi Júnior ${ }^{(4)}$, Clovis Artur Almeida da Silva ${ }^{(5)}$
}

\section{RESUMO}

Infecção pelo vírus varicela zoster (VVZ) em pacientes com lúpus eritematoso sistêmico juvenil (LESJ) tem sido pouco descrita. Durante um período de 12 anos, ocorreram 195 internações em 77 pacientes com LESJ e estas foram acompanhadas pela Unidade de Reumatologia Pediátrica do Instituto da Criança do Hospital das Clínicas da Universidade de São Paulo. Onze pacientes (14\%), dez do sexo feminino, apresentaram 14 internações $(7,1 \%)$ pelo VVZ. Nesses pacientes, a média de idade foi de 16 anos e 5 meses e a média do tempo de duração do LESJ até a primeira infecção devido ao VVZ foi de 4 anos. Todos os episódios das infecções estavam associados com atividade da doença, que se apresentaram como lesões vesicobolhosas seguindo trajeto nervoso. As regiões do tórax e membros foram mais comumente afetadas. Todos haviam utilizado prednisona e quatro usaram ciclofosfamida EV. Todos receberam aciclovir EV por 7 a 10 dias. Nenhum paciente apresentou neuralgia pós-herpética, infecção bacteriana secundária ou evoluiu para óbito. Entretanto, uma paciente em uso de aciclovir apresentou amaurose aguda por vasculite necrosante retiniana bilateral associado ao VVZ, necessitando de duas aplicações de ganciclovir intravítreo e gamaglobulina $\mathrm{EV}(2 \mathrm{~g} / \mathrm{kg} /$ dose $)$, com recuperação parcial da acuidade visual. Assim sendo, infecção por VVZ em pacientes com LESJ foi infreqüente, habitualmente associada à atividade da doença e à corticoterapia. Essa infecção foi controlada com aciclovir, e os pacientes raramente apresentaram complicações.

Palavras-chave: lúpus eritematoso sistêmico juvenil, corticosteróides, ciclofosfamida, infecção, vírus varicela zoster, aciclovir.

\begin{abstract}
Varicella zoster virus $(V Z V)$ infection in patients with juvenile systemic lupus erythematosus (JSLE) has been rarely described. 195 hospitalizations of 77 JSLE patients occurred in a period of 12 years and were followed at the Pediatric Rheumatology Unity of the Instituto da Criança - Hospital das Clínicas - Universidade de São Paulo. Eleven patients (14\%), 10 female, had 14 hospitalizations (7.1\%) due to $V Z V$. In these patients, the mean age was 16 years and 5 months and the mean duration of the JSLE until the first infection was 4 years. All of these VZV infections were associated with disease activity. All patients presented vesicles and blister lesions along a nerve. The thorax and limbs regions were the most frequently affected. All of the patients received prednisone and 4 cyclophosphamide IV. All patients received acyclovir $I V$ from 7 to 10 days. None of the patients had post-herpetic neuralgia, secondary bacterial infection or died. One patient that was receiving acyclovir had acute blindness by bilateral retinal necrosis vasculitis associated to the $V Z V$, needed two applications of intra-vitreous gancyclovir and immunoglobulin $(2 \mathrm{~g} / \mathrm{kg} /$ dose $I V)$ and her vision partially improved. VZV infection in patients with JSLE was rarely observed and was usually associated with disease activity and steroid use. Infection was controlled with acyclovir without serious adverse complications.
\end{abstract}

Keywords: juvenile systemic lupus erythematosus, corticosteroids, cyclophosphamide, infection, varicella zoster virus, acyclovir.

\footnotetext{
Recebido em 27/06/06. Aprovado, após revisão, em 21/09/06.

Unidade de Reumatologia Pediátrica e Comissão de Controle de Infecção Hospitalar do Instituto da Criança do Hospital das Clínicas da Faculdade de Medicina da Universidade de São Paulo (ICr-HC-FMUSP).

1. Aluna de graduação da FMUSP e bolsista da Iniciação Científica do Departamento de Pediatria da FMUSP.

2. Mestre em Ciências pelo Departamento de Pediatria da FMUSP.

3. Doutoras em Ciências pela FMUSP. Médicas-assistentes da Unidade de Reumatologia Pediátrica do ICr-HC-FMUSP.

4. Mestre em Medicina pela FMUSP. Responsável pela Comissão de Controle de Infecção Hospitalar do ICr-HC-FMUSP.

5. Professor colaborador e doutor em Medicina pela FMUSP. Responsável pela Unidade de Reumatologia Pediátrica do ICr-HC-FMUSP.

Endereço para correspondência: Dr. Clovis Artur Almeida da Silva, Rua Senador Cesar Lacerda Vergueiro, 494/82, CEP 05435-010, Vila Madalena, São Paulo, SP, Brasil, telefone: (11) 3069-8675, fax: (11) 3069-8510,e-mail: clovisaas@icr.hcnet.usp.br
} 


\section{INTRODUÇÃO}

Uma das principais causas de morbimortalidade no LESJ são as infecções bacterianas, virais, fúngicas, parasitárias ou causadas por protozoários ${ }^{(1,2)}$. Essas infecções estão, habitualmente, presentes nos pacientes com doença ativa, em uso de corticosteróides e imunossupressores, particularmente a pulsoterapia com ciclofosfamida endovenosa ${ }^{(3,4)}$.

O herpes zoster é causado pelo mesmo vírus responsável pela varicela $-\mathrm{o}$ vírus varicela zoster (VVZ) ou herpes vírus humano tipo 3 (HHV3) -, sendo resultado da reativação desse vírus latente ${ }^{(5)}$, podendo causar considerável morbidade em pacientes imunodeprimidos ${ }^{(6)}$. A causa dessa reativação é desconhecida, podendo estar relacionada a faixa etária, estresse ou imunodeficiências, tais como: tumores, síndrome da imunodeficiência adquirida (aids), doenças auto-imunes e uso de drogas imunossupressoras ${ }^{(7)}$.

A incidência de infecção pelo $\mathrm{VVZ}$ em pacientes adultos com LES varia de $3,2 \%$ a $43 \%^{(6-9)}$. Essa infecção está associada à atividade da doença e ao tratamento com ciclofosfamida endovenosa $^{(8)}$. Por sua vez, a infecção pelo VVZ em crianças e adolescentes com LESJ tem sido raramente descrita ${ }^{(2,3)}$.

A raridade de publicações avaliando essas infecções nos pacientes internados com LESJ e a expressiva casuística dessa doença no presente Serviço de Reumatologia Pediátrica estimularam a realização deste estudo.

Assim sendo, o objetivo foi descrever os episódios de infecções pelo VVZ em pacientes internados com LESJ e relacionar a presença dessas infecções com características clínicas e terapêuticas da doença.

\section{RELATO DE CASOS}

Durante o período de 12 anos (janeiro de 1994 a dezembro de 2005), 195 internações ocorreram em 77 pacientes com LESJ e foram acompanhadas pela Unidade de Reumatologia Pediátrica do Instituto da Criança do Hospital das Clínicas da Faculdade de Medicina da Universidade de São Paulo ICr-HC-FMUSP). Todos os pacientes preencheram os critérios de classificação para $\mathrm{O}$ diagnóstico de LESJ segundo o Colégio Americano de Reumatologia ${ }^{(10)}$ e apresentaram idade de início da doença inferior a 18 anos. O projeto de revisão retrospectiva de prontuários foi aceito pelo Comitê de Pesquisa e Ética do HC-FMUSP.

Os casos de lúpus internados com herpes zoster foram estudados com relação a dados demográficos, características clínicas, atividade da doença, curso, dano cumulativo, exames laboratoriais e terapias utilizadas para o LESJ no momento da infecção. A atividade do LESJ foi avaliada pelo Systemic Lupus Erythematosus Disease Activity Index

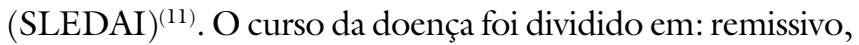
remissivo-recidivante e crônico ativo ${ }^{(12)}$. $\mathrm{O}$ dano cumulativo irreversível causado pelo LESJ e/ou pelo seu tratamento foi determinado pelo escore do Systemic Lupus International Collaborating Clinics/ACR (SLICC/ACR) Damage Index (SLICC-ACR-DI) ${ }^{(13)}$.

A Tabela 1 inclui dados demográficos, manifestações clínicas e tratamento de pacientes com LESJ e herpes zoster. Onze pacientes ( $14 \%$, dez do sexo feminino, apresentaram herpes zoster. Quatorze internações por infecção pelo VVZ $(7,1 \%)$ ocorreram nesses 11 pacientes; um paciente necessitou de duas (Caso 8) e outros de três hospitalizações (Caso 9), exclusivamente, por recidiva da infecção pelo VVZ.

A média de idade no momento da infecção pelo VVZ foi de 16 anos e 5 meses (variação de 12 a 20 anos) e a média do tempo de duração do LESJ até a primeira infecção pelo VVZ foi de 4 anos (variação de 5 meses a 7 anos e 9 meses) (Tabela 1 ).

Todos os pacientes apresentaram lesões vesicobolhosas sobre base eritêmato-edematosa que, habitualmente, seguiam um trajeto nervoso. As regiões do tórax e membros foram mais comumente afetadas. Esses pacientes foram hospitalizados e isolados, com precauções respiratórias (máscara) e de contato (avental e luvas).

Todos os episódios das infecções estavam associados à atividade da doença, com mediana do SLEDAI de 10 (variação de 5 a 28). A presença de algum dano cumulativo da doença (SLICC-ACR/DI $\geq 1$ ) foi evidenciada em três pacientes. Os cursos predominantes foram remissivorecidivantes em cinco e crônico ativo em quatro. Em dois pacientes, não foi possível a realização do curso, pois estes apresentavam menos de 6 meses de duração da doença. Portanto, nenhum dos pacientes teve curso remissivo. Envolvimento renal, confirmado por biópsia renal, ocorreu em dez casos: nefrite proliferativa difusa (classe IV) da Organização Mundial da Saúde (OMS) em quatro; nefrite proliferativa focal (classe III da OMS) em três; nefrite mesangial (classe II da OMS) em dois; e nefrite membranosa em um (Tabela 1). No momento da infecção pelo VHZ, todos estavam utilizando prednisona, quatro faziam pulsoterapia com ciclofosfamida endovenosa, dois utilizavam azatioprina e um, metotrexato (Tabela 1 ).

Para o tratamento da infecção pelo VVZ, todos receberam aciclovir $(30 \mathrm{mg} / \mathrm{kg} /$ dia a cada oito horas) endovenoso por 7 a 10 dias. Nenhum paciente apresentou nevralgia 
TABELA 1

DADOS DEMOGRÁFICOS, MANIFESTAÇÕES CLÍNICAS E TRATAMENTO DE PACIENTES COM LESJ E INFECÇÃO PELO VÍRUS VARICELA ZOSTER

\begin{tabular}{|c|c|c|c|c|c|c|c|c|c|}
\hline Caso & Sexo & Id(a) & $T p(a)$ & $\begin{array}{c}\text { SLEDAI / } \\
\text { SLICC } \\
\text { ACR/DI }\end{array}$ & Curso & $\begin{array}{l}\text { Manifestações } \\
\text { do LESJ }\end{array}$ & $\begin{array}{l}\text { Local } \\
\text { VVZ }\end{array}$ & $\begin{array}{l}\text { Trat. } \\
\text { LESJ }\end{array}$ & $\begin{array}{l}\text { Trat. } \\
\text { VVZ }\end{array}$ \\
\hline 1 & $\mathrm{~F}$ & 12 & 0,4 & $8 / N R$ & NR & Nefrite IV, convulsão & MSE, hemitórax E & $\mathrm{Pd}, \mathrm{MP}, \mathrm{PC}$ & Ac \\
\hline 2 & $\mathrm{~F}$ & 18 & 7,75 & $18 / 0$ & CA & Nefrite IV, vasculite & Lombar E & Pd & Ac \\
\hline 3 & $\mathrm{~F}$ & 17 & 0,8 & $12 / 0$ & RR & Nefrite III & Hemitórax E & $\mathrm{Pd}, \mathrm{PC}$ & Ac \\
\hline 4 & $\mathrm{~F}$ & 20 & 8,3 & $10 / 0$ & RR & Nefrite $V$ & MSE, hemitórax E & Pd, Aza & Ac \\
\hline 5 & $\mathrm{~F}$ & 16,5 & 2,6 & $8 / 0$ & $\mathrm{RR}$ & Nefrite II & Hemitórax E, região cervical & $\mathrm{Pd}$ & Ac \\
\hline 6 & $\mathrm{~F}$ & 12 & 1 & $8 / 1$ & CA & Vasculite & Hemitórax E & $\mathrm{Pd}, \mathrm{Cl}, \mathrm{Dap}$ & Ac \\
\hline 7 & $\mathrm{~F}$ & 18 & 1 & 12 / NR & NR & Nefrite III, artrite & MSD & $\mathrm{Pd}, \mathrm{Cl}, \mathrm{MTX}$ & Ac \\
\hline 8 & $\mathrm{~F}$ & 20 & 4,8 & $6 / 0$ & $\mathrm{RR}$ & Nefrite II & Face, MID e região lombar & $\mathrm{Pd} \mathrm{Cl}$ & Ac \\
\hline 9 & $\mathrm{~F}$ & 13 & 3 & $5 / 1$ & RR & Nefrite III & Hemitórax E & $\mathrm{Pd}$ & Ac \\
\hline 10 & $\mathrm{~F}$ & 19 & 12 & $28 / 1$ & CA & Nefrite IV & MSD, MID, tronco & $\mathrm{Pd}, \mathrm{PC}, \mathrm{Aza}$ & Ac, Gc \\
\hline 11 & M & 15 & 3 & $10 / 0$ & $\mathrm{CA}$ & Nefrite IV & MIE & $\mathrm{Pd}, \mathrm{PC}$ & Ac \\
\hline
\end{tabular}

$\mathrm{VVZ}=$ vírus varicela zoster; $\mathrm{F}=$ sexo feminino; $\mathrm{M}=$ sexo masculino; $\mathrm{Tp}=$ tempo de duração da doença até a infecção pelo VHZ; Id = idade da infecção pelo $\mathrm{VHZ}$; $\mathrm{a}=$ anos; Trat = tratamento; Loc = localização; LESJ = lúpus eritematoso sistêmico juvenil; SLEDAI = Systemic Lupus Erythematosus Disease Activity Index; SLICC-ACR/DI = Systemic Lupus International Collaborating Clinics/ACR Damage Index; $C A=$ curso crônico ativo; $R R=$ curso remissivo recidivante; $R=$ remissivo; $\mathrm{NR}=$ não realizado; $\mathrm{Pd}=$ prednisona; $\mathrm{Cl}=$ cloroquina; $\mathrm{PC}=$ pulsoterapia com ciclofosfamida; $\mathrm{Aza}=$ azatioprina; $\mathrm{MTX}=$ metotrexato; $\mathrm{Ac}=\mathrm{aciclovir} ; \mathrm{GC}=\mathrm{ganciclovir} ;$ $\mathrm{D}=$ direito; $\mathrm{E}=$ esquerdo; $\mathrm{MS}=$ membro superior; $\mathrm{MI}=$ membro inferior.

pós-herpética, infecção bacteriana secundária ou evoluiu para óbito após a infecção pelo VVZ. Uma paciente que utilizava dose alta de prednisona $(1,0 \mathrm{mg} / \mathrm{kg} / \mathrm{dia})$ recidivou três vezes da infecção pelo VVZ.

Entretanto, uma paciente (Caso 10) em uso de aciclovir endovenoso apresentou amaurose aguda por vasculite necrosante retiniana bilateral associado ao VVZ no terceiro dia de terapia antiviral, necessitando de duas aplicações de ganciclovir intravítreo e gamaglobulina endovenosa ( $2 \mathrm{~g} / \mathrm{kg} /$ dose $)$, com recuperação parcial da acuidade visual.

\section{DISCUSSÃO}

A infecção é uma das principais causas de morbimortalidade nos pacientes com LESJ. O aumento de infecção nestes pode ser decorrente do uso de terapêuticas imunossupressoras e da própria atividade da doença ${ }^{(1-3)}$. Por sua vez, a presença de alterações imunológicas próprias da doença, tais como disfunção de fagócitos, linfopenia, neutropenia, asplenia funcional, assim como diminuição de citosinas, imunoglobulinas e frações do complemento, predispõe ao desenvolvimento de infecções, como as virais pelo VVZ, apresentadas nos pacientes com LESJ desta casuística ${ }^{(2,14)}$.

Os pacientes com LESJ admitidos nas diversas enfermarias do ICr e incluídos neste estudo tinham o diagnóstico prévio da doença de base e eram seguidos no ambulatório. Todos os pacientes apresentaram varicela prévia e não foram vacinados anteriormente. Em virtude da imunossupressão pela própria doença e dos medicamentos utilizados para o
LESJ, nestes 12 anos avaliados, todos os pacientes ambulatoriais que tiveram infecção pelo VVZ foram internados e tratados com aciclovir endovenoso entre 7 e 10 dias. O aciclovir oral deve ser evitado para o tratamento da infecção pelo VVZ em crianças imunossuprimidas, por apresentar uma reduzida biodisponibilidade quando administrado por esta via com absorção de apenas $20 \%$ da droga ${ }^{(15)}$.

Depois de um episódio de varicela, o vírus em estado inativo reside nos tratos dos nervos espinhais. Por sua vez, quando reativado, este se espalha ao longo do nervo, podendo causar neurite (dor ou uma sensação de queimação). As erupções cutâneas aparecem em 2 a 3 dias após o vírus atingir a pele. Tais erupções costumam aumentar durante os próximos 3 a 5 dias, podendo evoluir para conteúdo purulento (pústulas) ou hemorrágico. Depois, as vesículas se rompem formando pequenas úlceras entre 2 e 3 semanas, que podem evoluir para cicatrizes ${ }^{(7)}$.

As lesões, habitualmente, aparecem ao longo de um ou três dermátomos e são unilaterais. $\mathrm{O}$ tronco é geralmente afetado, podendo ocorrer lesões em região cervical, face (particularmente ao longo do nervo trigêmeo) $)^{(7)}$. Nesta casuística, os locais mais freqüentemente afetados foram tronco e membros.

Infecções por VVZ em populações de LESJ têm sido raramente descritas. Em um estudo nacional, Len et $a l .^{(3)}$ diagnosticaram herpes zoster em $19 \%$ dos pacientes acompanhados com LESJ. Em outro estudo, Opastirakul e Chartapisak ${ }^{(2)}$ evidenciaram infecções pelo VVZ em $15 \%$ dos pacientes com LESJ em uso de ciclofosfamida oral 
e 5\% em uso de pulsoterapia com ciclofosfamida endovenosa. No presente estudo, todos os pacientes estavam em atividade da doença, recebiam corticosteróides (acima de $10 \mathrm{mg}$ de prednisona) e quatro estavam em vigência de pulsoterapia endovenosa com ciclofosfamida para nefrite lúpica e formas proliferativas (III ou IV).

Um aspecto relevante é que, na faixa etária pediátrica, excepcionalmente, há neuralgia pós-herpética ou outras complicações $^{(5)}$ e, geralmente, apresenta adequada resposta ao aciclovir, conforme foi evidenciado nos nossos casos.

Por sua vez, uma complicação rara e grave da infecção pelo VVZ foi evidenciada em um caso desta casuística. $\mathrm{Na}$ vigência de uma infecção ativa por VVZ e em vigência de aciclovir, uma paciente apresentou amaurose súbita com vasculite necrótica retiniana bilateral que evoluiu com controle parcial após gamaglobulina endovenosa e infusão de ganciclovir intravítreo. Essa complicação tem sido raramente relatada em pacientes adultos com LES e infecção pelo VVZ, com seqüela ocular definitiva habitualmente com redução importante da acuidade visual bilateral, conforme ocorreu naquela paciente ${ }^{(16,17)}$. Não há descrição dessa complicação em pacientes com LESJ.

Os pacientes com LESJ devem ser sempre vacinados, mas se apresentam ao reumatologista pediátrico em diferentes estados de proteção vacinal, geralmente incompleta, principalmente os adolescentes. Não existem, até o momento, recomendações específicas para os pacientes com doenças reumáticas auto-imunes e vacina do VVZ. Como

\section{REFERÊNCIAS}

1. Canova EG, Rosa DC, Vallada MG, Silva CA: Invasive aspergillosis in juvenile systemic lupus erythematosus. A clinicopathologic case. Clin Exp Rhematol 20: 736, 2002.

2. Opastirakul S, Chartapisak W: Infection in children with lupus nephritis receiving pulse and oral cyclophosphamide therapy. Pediatr Nephrol 20: 1750-5, 2005.

3. Len CA, Terreri MT, Hilário MO: Lúpus eritematoso sistêmico juvenil e infecção. Rev Bras Reumatol 42: 218-22, 2002.

4. Facó MM, Nukumizu LA, Moraes AJ, Barros PC, Troster EJ, Silva CA: Avaliação dos óbitos e necropsias em pacientes internados em um Serviço de Reumatologia Pediátrica por um período de 10 anos. Rev Bras Reumatol 45: 55-63, 2005.

5. Ragozzino MW, Melton LJ, Kurland LT, Chu CP, Perry HO: Population-based study of herpes zoster and its sequelae. Medicine (Baltimore) 61: 310-6, 1982.

6. Park HB, Kim KC, Park JH, et al: Association of reduced CD4 T cell responses specific to varicella zoster virus with high incidence of herpes zoster in patients with systemic lupus erythematosus. J Rheumatol 31: 2151-5, 2004. regra geral, os imunossuprimidos não devem receber vacinas com vírus vivos atenuados pelo risco de desenvolver a infecção ${ }^{(18)}$. Por sua vez, vacinação de familiares dos pacientes com LESJ deve ser encorajada ${ }^{(19)}$. Na Unidade de Reumatologia Pediátrica do ICr-HC-FMUSP, os pacientes com LESJ são vacinados, apenas antes (entre uma e duas semanas) da introdução dos corticosteróides e/ou imunossupressores, particularmente aqueles que não apresentaram varicela prévia. A eficácia e a segurança da vacina da varicela em pacientes com LESJ, em vigência de corticosteróides $\mathrm{e} /$ ou imunossupressores, ainda não foram estabelecidas.

Em resumo, infecção por VVZ em pacientes com LESJ foi raramente evidenciada, habitualmente associada à atividade da doença e ao uso de corticosteróides. Essa infecção foi adequadamente controlada com aciclovir e os pacientes raramente apresentaram complicações. $\mathrm{Na}$ presença de amaurose súbita, é imperativo o seguimento oftalmológico, com possibilidade da infusão de antivirais intravítreos, pelo risco de cegueira definitiva.

\section{AGRADECIMENTOS}

Aos médicos oftalmologistas da Disciplina de Oftalmologia da FMUSP e à Comissão de Infecção Hospitalar do ICr-HC-FMUSP. Este estudo foi patrocinado pelo Conselho Nacional de Desenvolvimento Científico e Tecnológico (CNPq) (número 302469/2005-2 para CAAS).

Declaramos a inexistência de conflitos de interesse.

7. Straus SE, Ostrove JM, Inchauspe G, et al: NIH conference. Varicella-zoster virus infections. Biology, natural history, treatment, and prevention. Ann Intern Med 108: 221-37, 1998. Review.

8. Moutsopoulos HM, Gallagher JD, Decker JL, Steinberg AD: Herpes zoster in patients with systemic lupus erythematosus. Arthritis Rheum 21: 789-802, 1978.

9. Nagasawa K, Yamauchi Y, Tada Y, Kusaba T, Niho Y, Yoshikawa $\mathrm{H}$ : High incidence of herpes zoster in patients with systemic lupus erythematosus: an immunological analysis. Ann Rheum Dis 49: 630-3, 1990.

10. Hochberg MC: Updating the American College of Rheumatology revised criteria for the classification of systemic lupus erythematosus. Arthritis and Rheumatism 40: 1725, 1997.

11. Brunner HI, Silverman ED, To T, Bombardier C, Feldman BM: Sensitivity of the Systemic Lupus Erythematosus Disease Activity Index, British Isles Lupus Assessement Group Index, and Systemic Lupus Activity Measure in the evaluation of clinical change in childhood-onset systemic lupus erythematosus. Arthritis and Rheumatism 42: 1354-60, 1999. 
12. Barr SG, Zonana-Nacach A, Magder LS, Petri M: Patterns of disease activity in systemic lupus erythematosus. Arthritis and Rheumatism 42: 2682-8, 1990.

13. Brunner HI, Silverman ED, To T, Bombardier C, Feldman BM: Risk factors for damage in childhood-onset systemic lupus erythematosus. Arthritis and Rheumatism 46: 436-4, 2002.

14. Greenberg SB: Infections in the immunocompromised rheumatologic patient. Crit Care Clin 18: 931-56, 2002.

15. Baldacci $\mathrm{E}$, Takanori PT: Infecção pelo vírus varicela zoster. In: Marcondes E, Vaz FAC, Ramos JLA, Okay Y. Pediatria Básica Tomo 2, 9a ed, São Paulo, Sarvier, 2003, pp. 137-40.
16. Matthews BN, Erb N, Gordon C, Callear AB, Murray PI, Salmon $\mathrm{M}$ : Unilateral varicella zoster virus ophthalmicus and contralateral acute retinal necrosis. Eye 16: 778-80, 2002.

17. Suraiya MS, Norazlina B, Carmen C, Muhaya M: Bilateral optic neuritis in pregnancy. Med J Malaysia 2003 58: 771-3, 2003.

18. Davies K, Woo P: British Paediatric Rheumatology Group. Immunization in rheumatic diseases of childhood: an audit of the clinical practice of British Paediatric Rheumatology Group members and a review of the evidence. Rheumatology 41: 937-41, 2002.

19. Douglas KM, Gordon C, Osman H, et al: Lupus and zoster. Lancet 23; 362: 616, 2003. 